\title{
Modified technique for tracheobronchial Y-stent insertion using flexible bronchoscope for stent guidance
}

\author{
Jordan Kazakov, MD, Mohamed Khereba, MD, Vicky Thiffault, RN, André Duranceau, MD, \\ Pasquale Ferraro, MD, and Moishe Liberman, MD, PhD, Montreal, Canada
}

From the Thoracic Surgery Department, Centre Hospitalier de L'Université de Montréal, Montreal, Canada.

Disclosures: Authors have nothing to disclose with regard to commercial support.

Received for publication March 20, 2015; revisions received May 24, 2015; accepted for publication July 3, 2015 ; available ahead of print Aug 12, 2015.

Address for reprints: Jordan Kazakov, MD, 11100 Euclid Ave, Cleveland, OH 44106 (E-mail: JordanKazakov@ gmail.com).

J Thorac Cardiovasc Surg 2015;150:1005-9

0022-5223/\$0.00

Published by Elsevier Inc. on behalf of The American Association for Thoracic Surgery

http://dx.doi.org/10.1016/j.jtcvs.2015.07.016

Airway obstruction may be caused by malignant and nonmalignant processes. The most common cause is bronchogenic carcinoma. Distal tracheal obstruction with or without involvement of the carina and the proximal main stem bronchi is an extremely challenging problem. Tracheobronchial silicone Y-stent insertion can be effective for both endoluminal obstruction and extrinsic airway compression and can achieve improvement of symptoms and quality of life. Y-stents also are effective in the treatment of tracheoesophageal fistula when it involves the distal trachea or carina.

Y-stents, although effective, can be difficult to place because of loss of the airway, inability to visualize the airway during stent placement, inability to ventilate during placement, and difficulty maneuvering the stent limbs into the correct position. These difficulties led us to develop a modification of the classic technique that addresses some of the challenges during the placement. We present the modified technique for Y-stent insertion using a combination of rigid and flexible bronchoscopy and review our initial experience and results.

\section{PATIENTS AND METHODS}

This study consists of a retrospective cohort including 29 consecutive patients with central airway obstruction treated with Y-stent insertion using the modified technique performed at the Centre Hospitalier de L'Université de Montréal Endoscopic Tracheobronchial and Oesophageal Center between October 2009 and September 2014. The patients were identified from a prospectively accrued interventional endoscopy research database.

The study was approved by the institutional review board of Centre de Recherche de L'Université de Montréal. Written informed consent was not required because of the retrospective nature of the study.

The patient characteristics, diagnosis, procedure characteristics, length of hospital stay, follow-up, and complications were evaluated using hospital charts and procedural records. We describe a modified technique for insertion of tracheobronchial Y-stents with Seldinger-like guidance over a flexible bronchoscope for Y-stent insertion and review the initial experience with this technique.

Insertion of Y-stents was performed using local and general anesthesia. General anesthesia was used for rigid bronchoscopy and

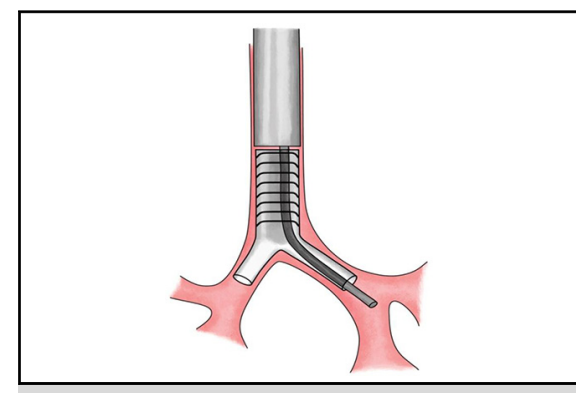

The flexible bronchoscope is used as a guide for the Y-stent similar to a guidewire.

Central Message

We describe a modified technique for Y-stent placement using a flexible bronchoscope for stent guidance in a Seldinger-like technique.

See Editorial Commentary page 1009.

placement of the stent through the larynx. Local anesthesia was used in selected cases in which a tracheostomy fistula was used for the insertion of the Y-stent.

The first phase of the procedure started with an evaluation of the airway and followed by treatment of endoluminal disease with any of the following techniques: mechanical debulking, laser ablation, electrocoagulation, and balloon dilatation. The next step was the measurement of the diameter and length of the trachea and both main stem bronchi. The diameter of the airways can be measured using the balloon of the radial ultrasound probe, which is inflated with water until it fills the whole airway, and the diameter of the balloon is measured on the ultrasound image. ${ }^{1}$ Another approach is to estimate the diameter of the airway from the diameter of the rigid and the flexible bronchoscopes after the ablative procedures are completed. The measurement allows for selection of the largest possible stent diameter to reduce the risk for stent obstruction and migration. We always measure the length of the left and right main stem bronchi and the length of the trachea involved with the disease. The length of the limbs should be long enough to cover the lesion and be as short as possible to decrease the chance for obstruction of the stent from secretions.

The second phase of the procedure involves insertion of the stent into the airway, advancing the stent distally and maneuvering the stent limbs into the correct position. A Macintosh (blade sizes 3 or 4) laryngoscope is used to visualize the vocal cords and guide the advancement of the stent placed over a rigid forceps (alligator forceps or Freitag forceps) between the cords into the proximal trachea (Figure 1).

Once the stent is deposited into the proximal trachea beneath the cricoid cartilage, the rigid forceps is withdrawn and a rigid tracheoscope or a rigid bronchoscope is inserted into the proximal trachea with the tip of the scope placed at the proximal end of the stent. The flexible bronchoscope is passed consecutively through the rigid tracheoscope (bronchoscope), the tracheal limb of the stent, and one of the bronchial limbs of the stent, and directed into the left or right lower lobe bronchus (Figure 2). A degree of 


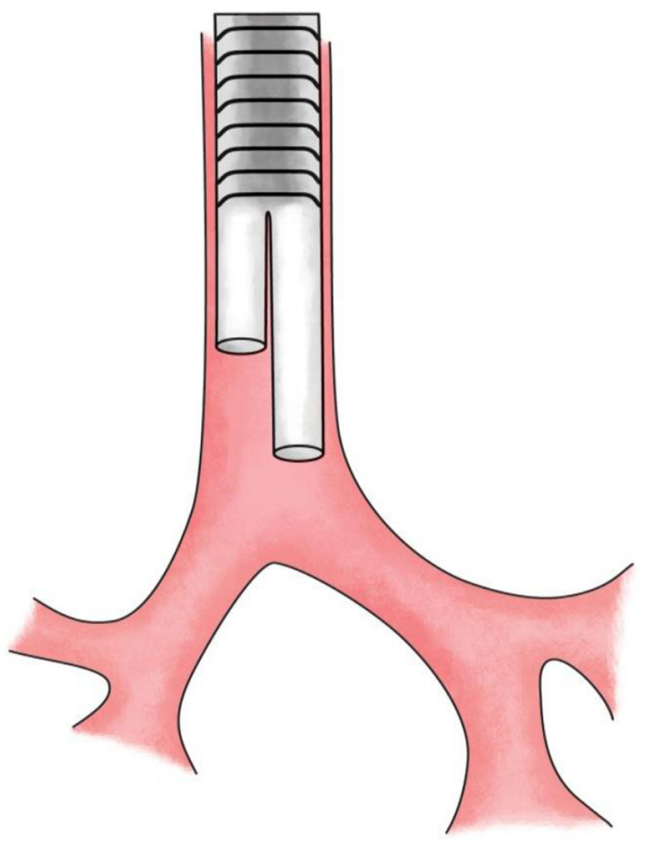

FIGURE 1. The Y-stent is placed in the proximal trachea, and the rigid scope is introduced in the trachea above the stent.

deformation of the limbs of the stent will be seen as they are compressed together to accommodate the stent in the trachea. Difficulty passing the flexible scope through the deformed limbs of the stent may be anticipated; however, we were able to pass a 5- to 6-mm flexible bronchoscope through the bronchial limb in all our cases. The flexible bronchoscope is passed through the limb of the stent corresponding to the bronchus that is more obstructed and has a more abnormal take-off from the carina. The rigid tracheoscope (bronchoscope) is then slowly advanced pushing the Y-stent

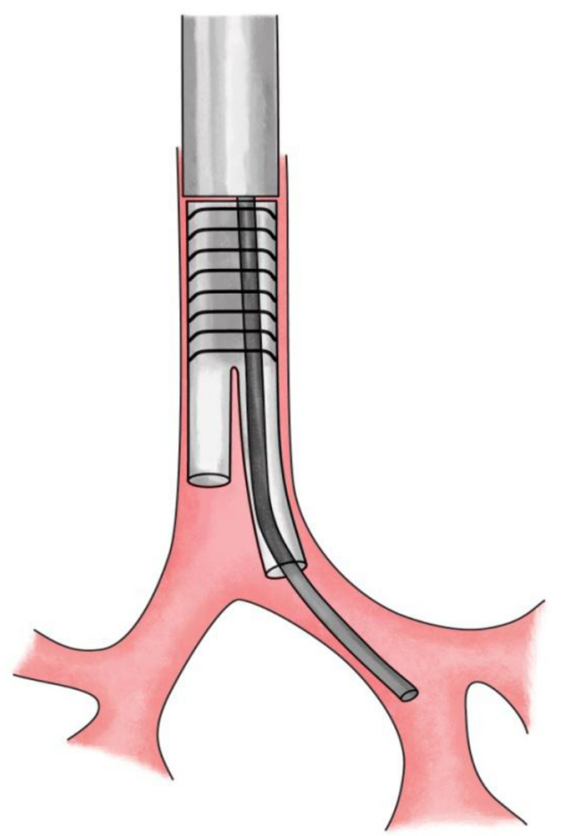

FIGURE 2. The flexible bronchoscope is passed down through the tracheoscope, tracheal limb, and one of the bronchial limbs of the Y-stent into the lower lobe bronchus.

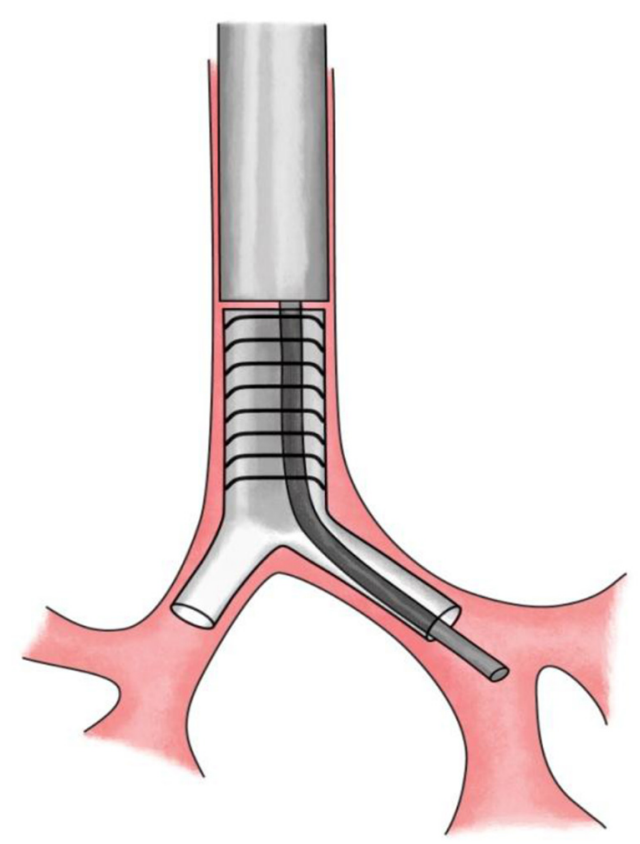

FIGURE 3. The rigid bronchoscope is advanced gently pushing the Y-stent over the flexible bronchoscope into position.

over the flexible bronchoscope using it as a guide for the stent's advancement in a bronchoscopic Seldinger technique (Figures 3 and 4). Intermittently, the advancement is held and the flexible bronchoscope is withdrawn proximally into the stent to check the position, help with orientation, and guide the progression of the stent limbs.

In patients with tracheostomy, the stent is inserted via the tracheal stoma and then the flexible bronchoscope is passed through the stent to the lower lobe bronchus. The stent is advanced distally using an approach similar to that described earlier, with the only difference being that a curved clamp is used to push the stent instead of the rigid scope.

\section{RESULTS}

A total of 29 patients underwent 35 procedures using the Seldinger technique for Y-stent insertion between October 2009 and September 2014 (Table 1). Patients' mean age was 61 years; the youngest patient was aged 34 years, and the eldest patient was aged 86 years. There were 16 female patients and 13 male patients. The most common presenting symptoms were dyspnea and stridor; other presenting symptoms were cough, hemoptysis, and infection. Tracheoesophageal fistula was present in 4 cases.

The obstruction was caused by endoluminal lesion or by external compression. The location of the disease is presented in Table 2. Malignancy, either primary pulmonary or metastatic, was the cause of the obstruction in 23 patients $(79 \%)$ (Table 3$)$. Non-small cell lung cancer was the most common malignant diagnosis (14 patients [48\%]), followed by esophageal cancer (5 patients $[17 \%])$. Tracheobronchomalacia was the most common diagnosis in the nonmalignant group; 1 patient had Y-stent placement for obstruction caused by relapsing polychondritis, and in 1 patient the Y-stent was placed for distal 


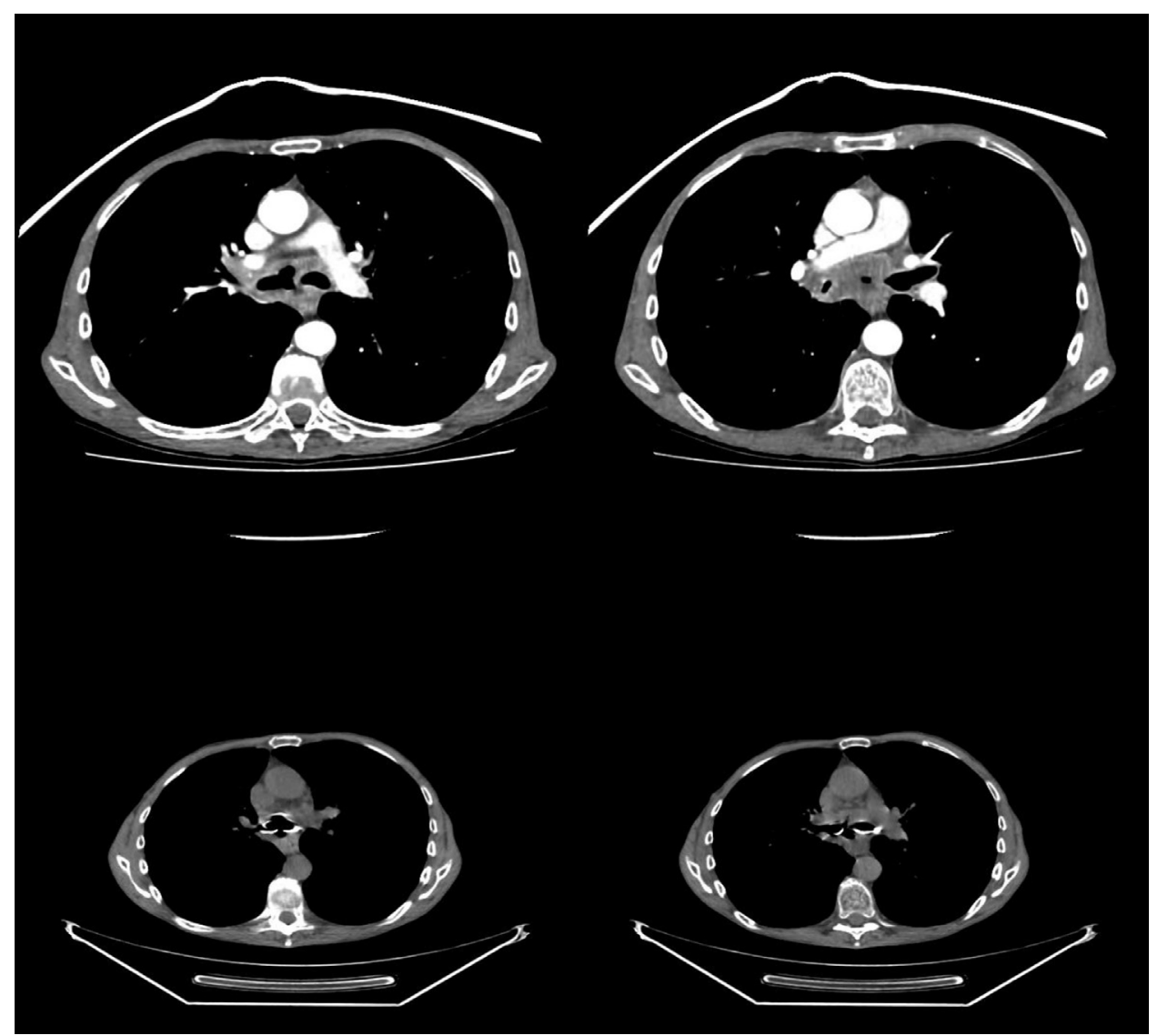

FIGURE 4. Computed tomography of the chest of a patient with a subcarinal mass and tracheomediastinal fistula. The top 2 images are before and the bottom 2 images are after the stent placement.

tracheal laceration extending to the right mainstem bronchus caused by endotracheal intubation. Thirty-four procedures were performed under general anesthesia, and 1 procedure was performed under moderate sedation with local anesthesia. The stent was placed in the trachea via the tracheal stoma in 3 patients, all of whom had severe tracheobronchomalacia.

The most commonly used stent was the Dynamic Y-Stent (Boston Scientific, Natick, Mass) with a tracheal limb width of 11 to $15 \mathrm{~mm}$. In 5 patients, the Hood Y-Stent (Hood Laboratories, Pembroke, Mass) with an outer diameter of $14 \mathrm{~mm}$ was used (Table 4).

The Seldinger technique was used without difficulty in 34 procedures $(97 \%)$ with satisfactory positioning of the stent

TABLE 1. Demographics

\begin{tabular}{lc}
\hline Procedures & 35 \\
Patients & 29 \\
Female & $16(55 \%)$ \\
Male & $13(45 \%)$ \\
Age, y (mean) & 61 \\
Minimum & 34 \\
Maximum & 86 \\
\hline
\end{tabular}

on the first attempt. The mean procedural time was $38 \mathrm{mi}-$ nutes (median time, 31 minutes). In 1 case, the endoscopist was unable to pass the flexible scope through the stent in a patient who had severe tracheobronchomalacia. The procedure was repeated at a later stage and was successful using the Seldinger technique for Y-stent placement.

No complications occurred during any of the procedures. All patients remained hospitalized for at least 24 hours after the procedure (median duration of stay, 2 days; range, 1110), and no immediate postprocedural complications were observed. The mean follow-up was 2.9 months; 2 patients were followed for 22 months. The most common late

TABLE 2. Location of lesion

\begin{tabular}{lc}
\hline \multicolumn{1}{c}{ Location of lesion } & No. $(\%$ of patients)** \\
\hline Trachea & $13(52 \%)$ \\
Carina & $6(24 \%)$ \\
Carina only & $3(12 \%)$ \\
Right main stem bronchus & $5(20 \%)$ \\
Left main stem bronchus & $6(24 \%)$ \\
Bilateral main stem bronchi & $7(28 \%)$ \\
\hline *Based on information from 25 patients (4 patients without detailed location descrip- \\
tion).
\end{tabular}


TABLE 3. Diagnosis

\begin{tabular}{lc}
\hline & No. $(\%)$ \\
\hline Malignant & \\
NSCLC & $14(48 \%)$ \\
Esophageal cancer & $5(17 \%)$ \\
SCLC & $1(3 \%)$ \\
Lymphoma & $1(3 \%)$ \\
Colon cancer & $1(3 \%)$ \\
Thymic cancer & $1(3 \%)$ \\
Nonmalignant & \\
Tracheobronchomalacia & $4(14 \%)$ \\
Relapsing polychondritis & $1(3 \%)$ \\
Intubation trauma & $1(3 \%)$ \\
\hline$N S C L C$, Non-small cell lung cancer; SCLC, small cell lung cancer. &
\end{tabular}

complication was obstruction of the stent from secretions, which required stent change in 4 patients. Another late complication was formation of granulation tissue at the proximal or distal ends of the stent.

Seven patients died within 4 weeks of the procedure. In 5 cases, the stent was removed between 1 and 6.5 months (mean, 2.4 months) after insertion. Eight patients had concomitant WallFlex esophageal stents (Boston Scientific) inserted for concurrent esophageal obstruction (4 patients) or tracheoesophageal fistula (4 patients) during the same procedure.

\section{DISCUSSION}

Therapeutic bronchoscopic interventions such as dilatation, laser photocoagulation, electrocoagulation, and mechanical debridement are used for the treatment of endoluminal bronchial obstruction. ${ }^{2}$ Stent placement is often needed to maintain airway patency after ablative procedures and in the treatment of airway obstruction from external compression. These techniques allow for successful withdrawal from mechanical ventilation and relief of symptoms. ${ }^{3,4}$

Y-stent insertion provides relief of the dyspnea and sustained improvement in quality of life for patients with central airway obstruction involving the carina, distal trachea, and proximal main stem bronchi. ${ }^{2,5}$ Y-stents are effective in restoring airway patency in both endoluminal obstruction and extrinsic compression of the airway.

TABLE 4. Stent types

\begin{tabular}{lc}
\hline & $\begin{array}{c}\text { No. (\% of successful } \\
\text { procedures) }\end{array}$ \\
\hline $\begin{array}{l}\text { Dynamic Y-Stent (Boston Scientific, Natick, } \\
\text { Mass) diameter }\end{array}$ \\
$15 \mathrm{~mm}$ & \\
$13 \mathrm{~mm}$ & $6(18 \%)$ \\
$11 \mathrm{~mm}$ & $16(47 \%)$ \\
Hood Y-Stent (Hood Laboratories, Pembroke, & $7(21 \%)$ \\
$\quad$ Mass) diameter & \\
$14 \mathrm{~mm}$ & $5(15 \%)$ \\
\hline
\end{tabular}

Y-stents, in combination with self-expanding esophageal stents, minimize the risk of recurrent infectious complications in patients with tracheoesophageal and bronchoesophageal fistulas. ${ }^{6}$ However, the insertion of a Y-stent can be technically challenging with the current techniques. Few techniques for Y-stent placement have been described. The classic method uses "push" or "pullback" techniques. The "push" technique uses alligator forceps to compress the bifurcation of the stent. The forceps and the stent are than advanced until reaching the distal trachea, just above the main carina where the forceps is slowly released, allowing the bronchial limbs to slide down in the appropriate main stem bronchi until the bifurcation of the stent reaches the carina. The forceps is then closed slightly and slowly withdrawn with care taken not to pull the stent out with the forceps. ${ }^{7}$ Another way of placement of the Y-stent in the airway includes "folding" of the Y-stent in an introducer tube that is passed down the rigid bronchoscope, and then with the help of a plunger the stent is pushed out of the stent introducer tube. With the "pullback" technique, both bronchial limbs of the stent are placed in one of the main stem bronchi and the stent is pulled until the shorter limb pops into position in the contralateral bronchus. ${ }^{8}$ The advancement of the stent can be guided fluoroscopically. The current techniques, although effective, require advancement of the Y-stent distally in the trachea and main stem bronchi without direct visualization of the airway. ${ }^{1}$ Blind advancement of the stent increases the risk of injury of the tracheal or bronchial wall and perforation of the membranous part of the trachea during the stent placement. The risk of airway perforation is further increased when there is malignant involvement of the airway wall and destruction of the main carina. Furthermore, in the presence of tracheoesophageal or tracheomediastinal fistula, the blind placement of the stent may lead to inadvertent advancement through the fistula. With our modification of the classic technique, the stent is guided over the flexible scope into position. Also, the ability to withdraw the flexible bronchoscope and assess the distal end of the stent limit the blind advancement of the stent to the initial deposition in the proximal trachea. The decreased "blind time" may decrease the chance for mechanical complications. To our knowledge, no previous data on the frequency of mechanical complications during Y-stent placement exist, but in our series there were no cases of perforation, extraluminal placement, and airway wall injury other than trivial abrasion. Further, no damage to the flexible bronchoscope was seen from the advancement of the stent.

The majority of patients who require Y-stent placement have poor respiratory reserve and extremely limited tolerance to hypoxia, which increases the procedure risk during the apnea time required for the Y-stent placement. Therefore, prompt placement of the stent and resumption of 
ventilation is of highest importance. The quick reintubation with the rigid tracheoscope (bronchoscope) after the stent deposition in the proximal trachea allows for secure airway with quick return of ventilation, and thus the apnea time is minimized compared with the classic techniques, in which the patient remains apneic until the stent is deposited in the distal trachea or proximal main stem bronchus.

The mean procedural time in our series was 38 minutes, which compares well with the procedure time reported by Oki and Saka. ${ }^{9}$ In their case series, they described a mean procedure time of 55 minutes for the conventional Y-stent technique. Thus, the procedural time using the modified technique was $31 \%$ less than the their procedural time with the conventional technique.

\section{CONCLUSIONS}

Our modification of the classic Y-stent insertion technique with Seldinger-like guidance over the flexible bronchoscope is safe, effective, expedient, and easy to master, and does not require new equipment. The advantages of this technique are visualization of the airway through most of the procedure, guided advancement of the stent, quick return of ventilation, and shorter procedural time.

\section{References}

1. Shirakawa T, Imamura F, Hamamoto J, Shirkakusa T. A case of successful airway stent placement guided by endobronchial ultrasonography. J Bronchol. 2004;11: 45-8.

2. Acuff T, Mack M, Ryan W. Simplified placement of a silicone tracheal Y stent Ann Thorac Surg. 1994;57:496-7.

3. Murgu S, Colt H. Silicone Y stent placement at secondary left carina for malignant central airway obstruction. J Thorac Cardiovasc Surg. 2010;139:494-5.

4. Dutau H, Toutblanc B, Lamb C, Seijo L. Use of the Dumon Y-stent in the manage ment of malignant disease involving the carina. Chest. 2004;126:951-8.

5. Colt HG, Harrell JH. Therapeutic rigid bronchoscopy allows level of care change in patients with acute respiratory failure from central airways obstruction. Chest. 1997;112:202-6.

6. Cavaliere S, Venuta F, Foccoli P, Toninelli C, La Face B. Endoscopic treatment of malignant airway obstructions in 2,008 patients. Chest. 1996;110:1536-42.

7. Shiraishi T, Kawahara K, Shirakusa T, Inada K, Okabayashi K, Iwasaki A. Stenting for airway obstruction in the carinal region. Ann Thorac Surg. 1998;66:1925-9.

8. van den Bongard HJ, Boot H, Bass P, Taal B. The role of parallel stent insertion in patients with esophagorespiratory fistulas. Gastrointest Endosc. 2002;55:110-5.

9. Oki M, Saka H. Double Y-stenting for tracheobronchial stenosis. Eur Respir J. 2012;40:1483-8.

\title{
EDITORIAL COMMENTARY
}

\section{Pushing, pulling, or Seldinger technique: What matters is understanding the principles, not the methods}

\author{
Erik Folch, MD, Adnan Majid, MD, and Sidhu P. Gangadharan, MD
}

\footnotetext{
From the Division of Thoracic Surgery and Interventional Pulmonology, Beth Israel Deaconess Medical Center, Harvard Medical School, Boston, Mass.

Disclosures: Authors have nothing to disclose with regard to commercial support.

Received for publication July 22, 2015; accepted for publication July 24, 2015; available ahead of print Aug 14, 2015.

Address for reprints: Sidhu P. Gangadharan, MD, 185 Pilgrim Rd, W/DC 201, Boston, MA 02215 (E-mail: sgangadh@bidmc.harvard.edu).

J Thorac Cardiovasc Surg 2015;150:1009-10

$0022-5223 / \$ \$ 36.00$

Copyright (C) 2015 by The American Association for Thoracic Surgery

http://dx.doi.org/10.1016/j.jtcvs.2015.07.071
}

As has been clearly stated, "As to methods, there may be a million and then some, but principles are few. The man who grasps principles can successfully select his own methods. The man who tries methods, ignoring principles, is sure to have trouble."

In this issue of the Journal, ${ }^{1}$ Kazakov and colleagues describe their method of placing tracheobronchial Y stents for benign and malignant airway disease in 29 patients with very good results and possibly less time. The limited literature on the topic of Y-stent placement is a surrogate marker

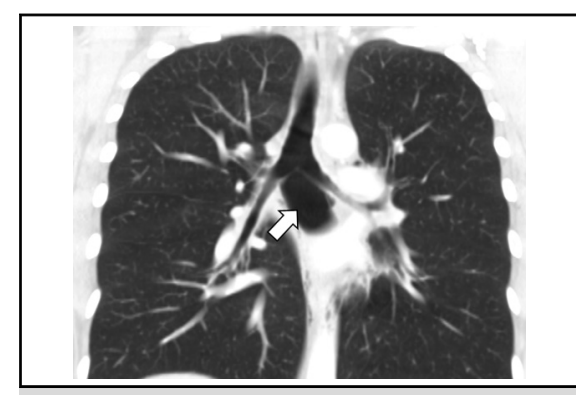

Coronal computed tomography showing mediastinal air from tracheal rupture during $\mathrm{Y}$-stent placement (arrom)

\section{Central Message}

Tracheobronchial stenting may result in potentially significant complications. Mastery of safe technique for placement is essential.

See Article page 1005 . 